\title{
Study on the Law of Pseudo-Cavitation on Superhydrophobic Surface in Turbulent Flow Field of Backward-Facing Step
}

\author{
Xuecheng Lv ${ }^{1}{ }^{1}$, Wei-Tao $\mathrm{Wu}^{2}{ }^{2}, \operatorname{Jizu}_{\mathrm{Lv}}{ }^{1}$, $\mathrm{Ke} \mathrm{Mao}^{1}{ }^{1}$, Linsong Gao ${ }^{1}$ and Yubai Li ${ }^{1, *}$ \\ 1 School of Energy and Power Engineering, Dalian University of Technology, Dalian 116024, China; \\ xc.1@mail.dlut.edu.cn (X.L.); lvjizu@dlut.edu.cn (J.L.); 190553957@mail.dlut.edu.cn (K.M.); \\ gaolinsong@mail.dlut.edu.cn (L.G.) \\ 2 School of Mechanical Engineering, Nanjing University of Science and Technology, Nanjing 210094, China; \\ weitaowwtw@njust.edu.cn \\ * Correspondence: liyubai@dlut.edu.cn
}

Citation: Lv, X.; Wu, W.-T.; Lv, J.;

Mao, K.; Gao, L.; Li, Y. Study on the

Law of Pseudo-Cavitation on

Superhydrophobic Surface in

Turbulent Flow Field of

Backward-Facing Step. Fluids 2021, 6,

200. https://doi.org/10.3390/

fluids 6060200

Academic Editor: Faik Hamad

Received: 21 April 2021

Accepted: 23 May 2021

Published: 27 May 2021

Publisher's Note: MDPI stays neutral with regard to jurisdictional claims in published maps and institutional affiliations.

Copyright: (c) 2021 by the authors. Licensee MDPI, Basel, Switzerland. This article is an open access article distributed under the terms and conditions of the Creative Commons Attribution (CC BY) license (https:/ / creativecommons.org/licenses/by/ $4.0 /)$.

\begin{abstract}
Superhydrophobic surface is regarded as important topic in the field of thermal fluids today due to its unique features on flow drag reduction and heat transfer enhancement. In this study, the pseudo-cavitation phenomenon on the superhydrophobic surface in the backward-facing step turbulent flow field is observed through experiments. The underlying reason for this phenomenon is studied with experimental observation and analysis, and the time variant mechanisms of this phenomenon with various Reynolds number is summarized. The research results indicate that the superhydrophobic surface and the backward-facing step provide the material basis and dynamic condition for the generation of pseudo-cavitation. The pseudo-cavitation induces a large bubble on the superhydrophobic surface below the backward-facing step. The size, position, shape, oscillation amplitude, detachment, and splitting of the large bubble show regularity with the changes of Reynolds number. Meanwhile, the bubble growth, oscillation, detachment, split, and regeneration over time also show regularity. The study of bubble generation and development laws can be used to better control the perturbation of the flow field. Importantly, the present study has meaning in better understanding the flow mechanisms and gas coverage of superhydrophobic surface under condition of backward-facing step, paving the way for studying the flow drag reduction effect of superhydrophobic surface.
\end{abstract}

Keywords: superhydrophobic surfaces; pseudo-cavitation; bubble; turbulence; drag reduction; enhanced perturbation

\section{Introduction}

Inspired by the super-hydrophobic characteristics of certain animal and plant surfaces from the nature, researchers began to explore the characteristics of super-hydrophobic surfaces in terms of anti-frost, self-cleaning, anti-corrosion, and fluid drag reduction. Generally, a surface with a static contact angle greater than $150^{\circ}$ and a rolling angle less than $10^{\circ}$ is called a superhydrophobic surface [1]. Today, worldwide research efforts have made significant progress in adopting superhydrophobic surfaces to the field of thermal fluids. For the theoretical aspect, Young [2] first proposed the mathematical formula to calculate the contact angle. In the following studies, the Wenzel model was proposed [3], as illustrated by Figure 1a, and the Cassie-Baxter model [4] was proposed, as illustrated by Figure 1b, for calculating the contact angle. Improvements have been made on these two classic models with accounting the new wetting states and their mathematical models [5,6]. Jiang et al. [7] summarized and further extended the descriptions of the superhydrophobic state. The pointed out that the states of superhydrophobic surface includes the Wenzel state, Cassie state, Gecko state, and the transition state between Wenzel and Cassie, as well as the Lotus state. The lotus state is a special case of the Cassie state, as shown in Figure 1c. The superhydrophobic surface prepared in this study can be 
categorized into the Lotus state, with a contact angle of $156^{\circ}$ and a contact angle hysteresis of $5^{\circ}\left(\theta_{A}-\theta_{R}\right)$, as shown in Figure $1 \mathrm{~d}-\mathrm{f}$. In recent years, the preparation method of superhydrophobic surface has been developing fast. The methods can be summarized into two categories [8]. First, some small-scale rough structures are fabricated on the substrate of hydrophobic materials. Second, coating low surface energy substances on the surface of materials with micro-nano structure.

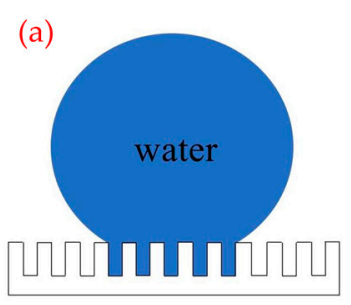

(d)

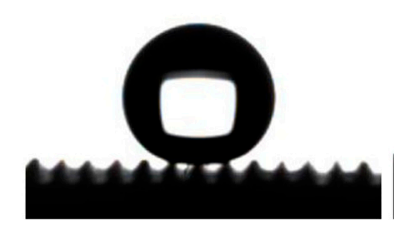

(b)

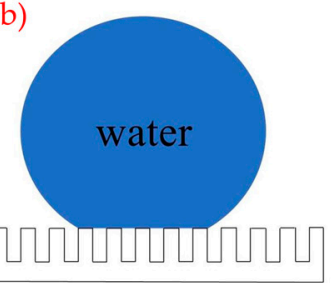

(e)

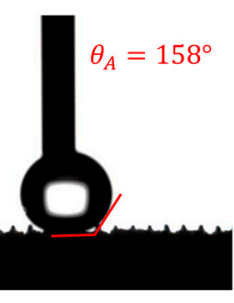

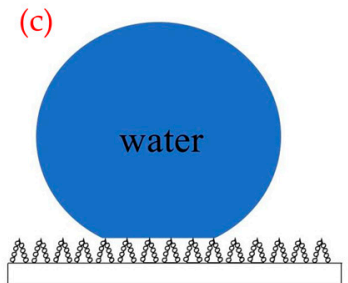

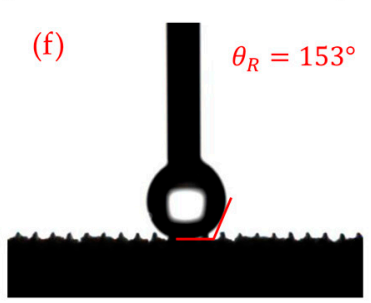

Figure 1. Wetting state: (a) Wenzel state; (b) Cassie-Baxter state; (c) Lotus state (a special case of the Cassie-Baxter state); $(\mathbf{d})$ contact angle hysteresis $\left(\mathrm{CA}=156^{\circ}\right)$ of water $(2 \mu \mathrm{L})$ droplet on the prepared superhydrophobic surface; (e) the advancing contact angle $\left(\theta_{A}=158^{\circ}\right)$ of water $(2 \mu \mathrm{L})$ droplet on the prepared superhydrophobic surface is measured by increasing the droplet volume. (f) The receding contact angle $\left(\theta_{R}=152^{\circ}\right)$ of water $(2 \mu \mathrm{L})$ droplet on the prepared superhydrophobic surface is measured by decreasing the droplet volume. The difference between the advancing and receding contact angles is particularly small, and the contact angle hysteresis $\left(\theta_{A}-\theta_{R}\right)$ is always less than $10^{\circ}$.

Many studies have shown that the superhydrophobic surface embraces the characteristics of drag reduction. The Cassie-Baxter model is widely used to explain the physical mechanisms of superhydrophobic surfaces with micro-nano structures [9]. Under the effect of surface tension, when the fluid flows through the superhydrophobic surface, it cannot completely remove the gas between the micro-nano structures on the surface, thus forming a gas-liquid interface and reducing the overall viscous resistance of the wall. Wang et al. [10] proposed that the mechanism of drag reduction on superhydrophobic surfaces is the boundary slip of the fluid due to the air trapped in the microstructure and demonstrated experimentally that the optimal drag reduction can be up to $13 \%$. Martell et al. [11] studied the drag reduction effect of superhydrophobic surface in turbulent flow via numerical simulation and found that the solid-liquid interface on the superhydrophobic surface had no slipping characteristics, while there was slipping at the gas-liquid interface. The slipping velocity of the superhydrophobic surface was as high as $75 \%$ of the mainstream average velocity, and the maximum expected drag reduction was up to $40 \%$. Park [9] studied the drag reduction of smooth surface and superhydrophobic surface by experiments. It was found that friction resistance of the super-hydrophobic surface decreased by 75\% compared with the smooth surface. With increasing of the gasliquid interface area, the friction resistance of superhydrophobic flow decreases. However, the super-hydrophobic surface will weaken the convective heat transfer, while reducing the resistance. Cowley et al. [12] studied the convection of superhydrophobic surface by numerical simulation. By assuming that the heat flux of solid-liquid interface of superhydrophobic surface is constant and the gas-liquid interface is adiabatic, the convective heat transfer of superhydrophobic surface is found to be less than that of regular smooth surface. Fuaad and Prakash [13] studied the effects of superhydrophobic surface on turbulent convective heat transfer by direct numerical simulation (DNS) method and found that the 
normal heat flux of superhydrophobic wall was smaller than that of smooth wall, and the Nusselt number of superhydrophobic surface was smaller than that of regular smooth wall.

In this study, the influence of superhydrophobic surface on the turbulent flow field is studied by creating the turbulent flow in a short pipeline through the backward-facing step structure. The backward-facing step structure has a wide range of applications in engineering, such as airfoils at large attack angle, spoiler flows, flow inside a combustor/condenser, flows in an enlarged pipe or a channel with steps, flows around a boat or a building, etc. $[14,15]$. The phenomenon of pseudo cavitation on the superhydrophobic surface downstream of the backward-facing step was studied. It is found a large stable bubble appeared on the superhydrophobic surface in the turbulent field, which showed regularity with the change of Reynolds number and time. The study of bubble generation and development laws can be used to better control the perturbation of the flow field. In addition, the present study has meaning in better understanding the flow mechanisms of superhydrophobic surface under condition of backward-facing step, paving the way for studying the flow drag reduction effect of superhydrophobic surface. And the flow resistance reduction and heat transfer enhancement effects corresponding to the backward-facing step flow will be focus of the future studies.

\section{Materials and Methods}

\subsection{Backward-Facing Step Flow Visualization Experimental Setup}

The experiment was carried out in the closed system setup shown in Figure 2, mainly including the intake tank, return tank, water pump, rotor flowmeter, development section, test section, and test pipeline. The system adopts self-circulation mode, and the circulation direction is counterclockwise. The working fluid in the backwater tank is recycled back to the backwater tank through the pump, rotor flowmeter, inlet tank, and experimental section. The flow channel in the development section is $5 \mathrm{~mm}$ high, $10 \mathrm{~mm}$ wide, and $1200 \mathrm{~mm}$ long, which ensures that the fluid in the test section is fully developed. The overflow plate is set in the intake tank, which plays a role in filtering and stabilizing the flow. In the experiment, two parallel rotor flowmeters were used to measure the large flow range $(40 \sim 400 \mathrm{~L} / \mathrm{h})$ and small flow range $(4 \sim 40 \mathrm{~L} / \mathrm{h})$. The water temperature of the circulatory system is controlled to be $20^{\circ} \mathrm{C}$.

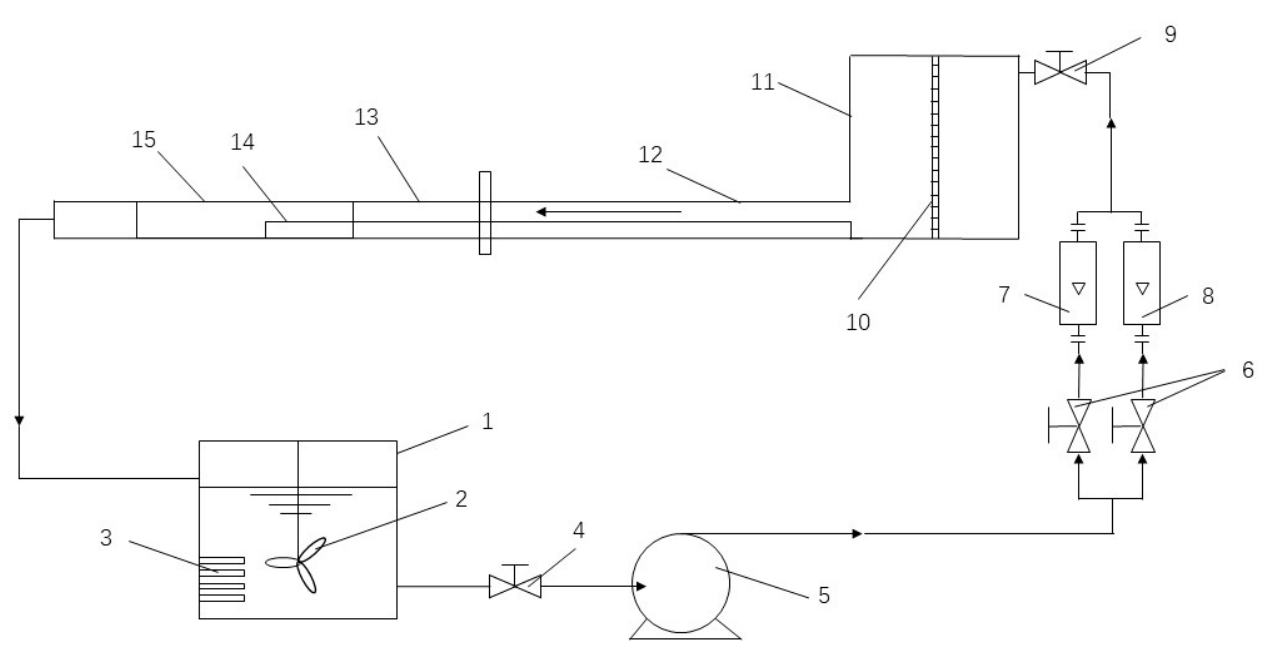

Figure 2. Schematic diagram of experimental system. 1: Reclaimer; 2: mixer; 3: constant temperature heater; 4,6,9: valve; 5: water pump; 7: large flow rotor flowmeter; 8: small flow rotor flowmeter; 10: overflow plate; 11: intake tank; 12: development section; 13: test section; 14: step; 15: quartz glass window. 


\subsection{Test Section Design and Surface Preparation}

The skeleton of the test section is made of aluminum, and its flow channel is of rectangular shape with a length of $430 \mathrm{~mm}$. As shown in Figure 3a, the height of the steps in the pipe is $5 \mathrm{~mm}$, the position is $150 \mathrm{~mm}$ from the entrance of the test section, and the expansion ratio of the pipe is $H / h=2: 1$, where $H$ is the height of the pipe, and $h$ is the height of the step. The cross-section of the flow channel above the steps is $10 \mathrm{~mm} \times 5 \mathrm{~mm}$, and the cross-section of the flow channel below the steps is $10 \mathrm{~mm} \times 10 \mathrm{~mm}$. As shown in Figure 3b, holes were machined on three sides of the tube wall $150 \mathrm{~mm}$ away from the entrance of the test section, and a transparent quartz glass observation window is embedded into it, which is used for high-speed camera shooting and LED lighting. A $150 \mathrm{~mm} \times 10 \mathrm{~mm}$ through hole was opened on the lower wall surface at $250 \mathrm{~mm}$ of the entrance of the test section to replace the lower bottom surface. After the quartz glass window and the replaceable bottom surface are installed, the clamps are used to fix the test section, and the sealant was evenly coated on the gap to maintain the tightness of the pipeline. Thread holes were machined $30 \mathrm{~mm}$ from the inlet and outlet of the test section to connect the pressure difference indicator. In this experiment, Reynolds number $\operatorname{Re}=U_{0} h / v_{0}$, where $h$ is the height of the step, $U_{0}$ measured by the flow meter is the average velocity of the flow channel upstream of the step, and $v_{0}$ is the kinematic viscosity coefficient. In the experiment, the water temperature was kept at $20^{\circ} \mathrm{C}\left(\right.$ At $\left.20{ }^{\circ} \mathrm{C}, v_{0}=1.006 \times 10^{-6} \mathrm{~m}^{2} / \mathrm{s}\right)$.
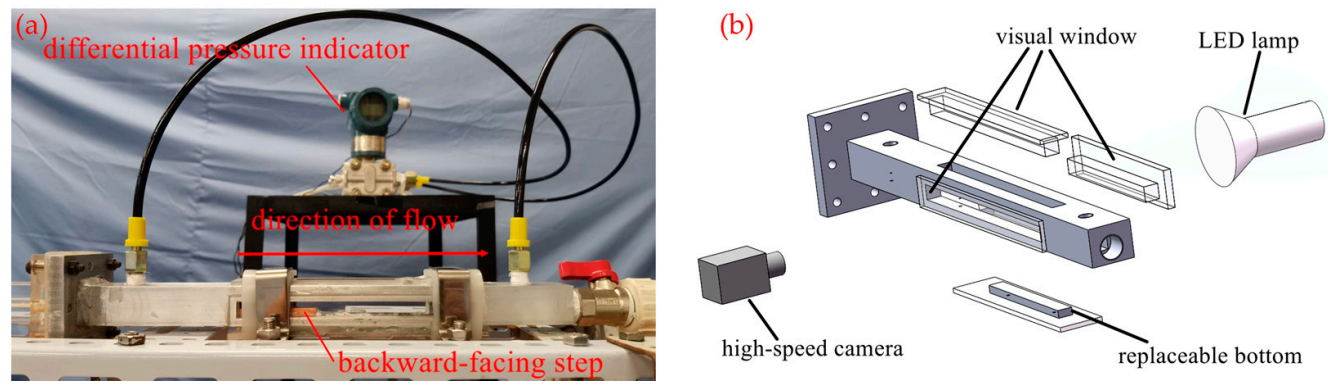

Figure 3. Test section. (a) Physical map; (b) Schematic diagram.

In the experiment, the average velocity and Reynolds number in the pipe are obtained by measuring the flow rate. The Reynolds number range is $2300 \sim 11,000$, the flow rate in the pipe above the step ranges from $0.46 \mathrm{~m} / \mathrm{s}$ to $2.21 \mathrm{~m} / \mathrm{s}$ and the corresponding flow rate range is $83.3 \sim 398 \mathrm{~L} / \mathrm{h}$. The image capture system consists of a high-speed camera (Revealer, 5KF10) and LED lamp. In the experiment, the exposure time of the high-speed camera is set to $200 \mu \mathrm{s}$, and the original frame rate is set to $2000 \mathrm{fps}$.

The bottom surfaces of several replaceable copper were machined by wire cutting and polished. The contact angle of the bottom surface of copper can reach about $87^{\circ}$, which is a hydrophilic surface, as shown in Figure $4 \mathrm{~b}$. And then the surfaces with different wettability are prepared on it. The copper substrate was cleaned by ultrasonic and then immersed in $30 \%$ hydrogen peroxide solution for $8 \mathrm{~h}$ to obtain a super hydrophilic surface with a static contact angle of $5^{\circ}$, as shown in Figure 4a. After ultrasonic cleaning, the copper substrate was sprayed with hydrophobic coating type 316,304 made by Shanghai Jie Dun Nano Technology Company to obtain a hydrophobic surface with a static contact angle of $120^{\circ}$, as shown in Figure $4 \mathrm{c}$.

(a)

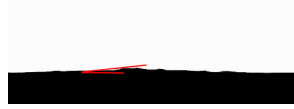

(b)

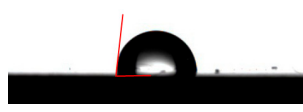

(c)

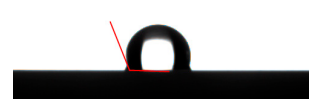

(d)

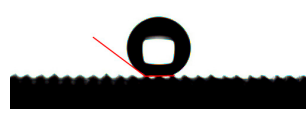

Figure 4. Contact angle characterization of each surface at room temperature, where the volume of the droplet is $2 \mu \mathrm{L}$. (a) Super hydrophilic surface; (b) hydrophilic surface; (c) hydrophobic surface; (d) superhydrophobic surface. 
Micro-nano structures were fabricated on the polished copper surface by laser processing. Figure 5 shows the scanning electron microscope (SEM) images of the micro-nano structure surface. It can be seen that uniform micro-columns are distributed on the copper surface. The size of the micro-column is $100 \mu \mathrm{m} \times 100 \mu \mathrm{m}\left(l_{1} \times l_{2}\right)$, and the interval between the micro-columns is $100 \mu \mathrm{m}\left(l_{3}\right)$, as shown in Figure $5 \mathrm{~b}$. Then, the NC319 superhydrophobic coating made by Changzhou Nanocoating Company was sprayed on the surface of micro-nano structure to make it a stable superhydrophobic surface. After spraying the superhydrophobic coating on the surface, bake in a vacuum drying oven at $70{ }^{\circ} \mathrm{C}$ for $20 \mathrm{~min}$. The static contact angle can reach $156^{\circ}$, as shown in Figure $4 \mathrm{~d}$.
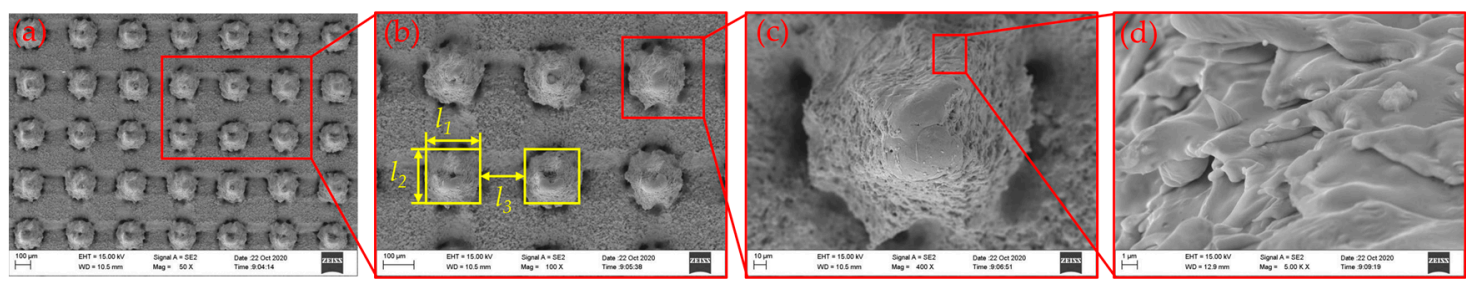

Figure 5. Scanning electron microscope (SEM) images of micro-nano structure: (a) 50 times magnification; (b) 100 times magnification; (c) 400 times magnification; (d) 5000 times magnification.

\subsection{Data Processing}

MATLAB software was used to post-process the images collected by the high-speed camera for batch processing, and the programmed MATLAB code can accurately capture the edge of the image and obtain the pixel information of the image.

The pictures captured by the high-speed camera (e.g., Figure 6a) often have problems, such as more background information, unclear bubble edges, inconspicuous contrast, and uneven background. To prevent these problems, the original image is first preprocessed by subtracting the background and grayscale processing, as shown in Figure 6b. Then, the image is binarized, as in Figure 6c. Finally, the Sobel image edge detection algorithm is used to extract the edges of the bubbles and fill the holes in the middle of the bubbles due to bright spots to obtain Figure $6 \mathrm{~d}$. By repeating the above steps, the contour and center of mass of the bubble at each moment can be identified, and the spatial distribution information of the bubble is obtained after comparing with the scale calibration information.

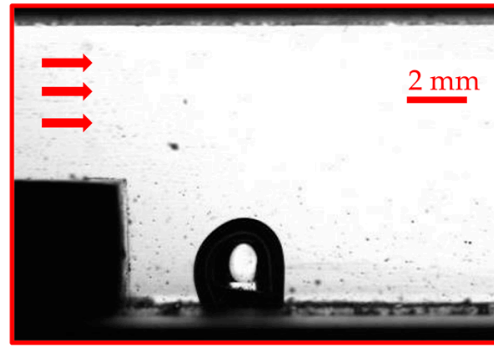

(a)

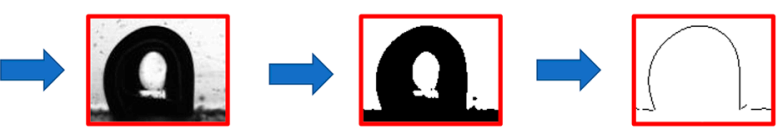

(b) (c) (d)

Figure 6. Image processing process (Take the picture under the typical working condition of $\operatorname{Re}=4000$ as an example.). (a) Original image; (b) background subtraction and grayscale processing; (c) binarization process; (d) edge detection and image fill.

Liu [16] et al. conducted an image processing study on bubbles obtained from twodimensional photography and showed that the bubble data obtained from two-dimensional photography are reasonably correct with an error of only $10 \%$ compared to the real data. For the bubbles attached to the wall, researchers often use the maximum width and maximum length of the bubble to calculate the equivalent diameter of the bubble [16-19]. As shown 
in Figure 7, after identifying the major axis $w$ and the minor axis $h$ of the bubble, the equivalent diameter and aspect ratio of the bubble can then be calculated.

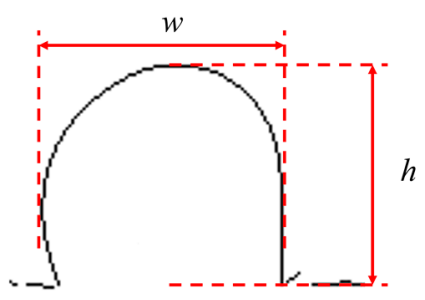

Figure 7. Diagram of the maximum width and length of the air bubble.

The bubble equivalent diameter is defined as $d_{e}$, which can quantify the volume of the bubble.

$$
d_{e}=\sqrt[3]{w^{2} h}
$$

In this paper, the range of bubble equivalent diameter is from 1 to $3 \mathrm{~mm}$. Liu et al. [16] evaluated the error of this method of describing the equivalent diameter. According to the evaluation results, the maximum error is $4 \%$, and the minimum error is $0.45 \%$ when using this method to evaluate the volume of bubbles in the range of $1 \sim 3 \mathrm{~mm}$ equivalent diameter, and the data are reasonable and correct.

Aspect ratio is then defined as $E$, which can indicate the degree of deformation of the bubble.

$$
E=\frac{h}{w}
$$

Determining the position of the bubble requires estimating the center of mass for the bubble, which can be calculated by the following formula:

$$
\begin{aligned}
& X=\frac{1}{N} \sum_{i=0}^{N-1} x_{i}, \\
& Y=\frac{1}{N} \sum_{i=0}^{N-1} y_{i} .
\end{aligned}
$$

In the formula, $x_{i}$ and $y_{i}$ are all the pixel coordinates in the bubble contour, and $X$ and $Y$ are the calculated bubble centroid coordinates. And $N$ is the total number of pixel points in the bubble outline in a frame.

\section{Results and Discussion}

\subsection{Experimental Observation}

Firstly, high-speed camera is used to photograph the surface through the glass window at the top of the channel to investigate the bubble attachment phenomenon on different wettability surfaces in the turbulent flow field downstream of the backward-facing step, as shown in Figure 8. On the super hydrophilic surface (Figure 8a), the attached bubbles are hardly seen and small, and the small bubbles are basically gathered on the surface of the corner vortex region beside the backward-facing step. On the hydrophilic surface (Figure $8 b$ ), a large number of bubbles with small volume are seen. Most of the bubbles are distributed in the recirculating flow region, and the volume of bubbles on the surface of the corner vortex region is slightly larger than that in other regions. On the hydrophobic surface (Figure 8c), the bubbles are mostly distributed on the surface of the recirculating flow region. Several large bubbles are gathered in the corner vortex region beside the backward-facing step, and the bubbles in other regions are small. On the superhydrophobic surface (Figure $8 \mathrm{~d}$ ), only a very large bubble appeared on the surface of the recirculating flow region. 

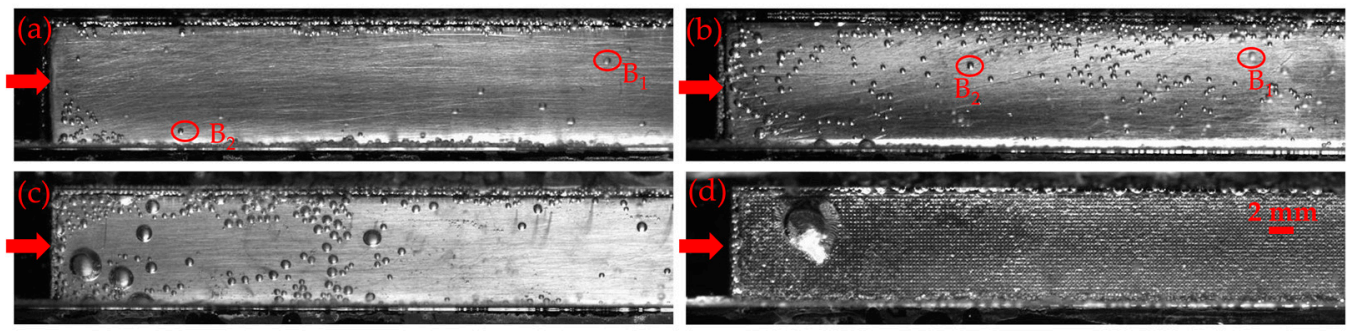

Figure 8. Bubble attachment on different surfaces downstream of the backward-facing step when the time is $88 \mathrm{~s}$. (a) Super hydrophilic surface; (b) hydrophilic surface; (c) hydrophobic surface; (d) superhydrophobic surface.

It is worth noting that the quartz glass window with a static contact angle of $52^{\circ}$ also has some bubbles adhering to the surface at a lower Reynolds number. However, the transparency of the bubbles attached on the surface of the glass window $\left(\mathrm{B}_{1}\right)$ and the bubbles attached on the copper surface $\left(B_{2}\right)$ are different and can be distinguished, as shown in Figure 8a,b. Since there are more bubbles attached to the glass window at low Reynolds number, the top view at the Reynolds number of 5000 working condition is selected for comparison. In addition, the direction indicated by the arrow in Figure 8 is the mainstream direction.

In the experiment, the Reynolds number in the channel is adjusted by controlling the flow velocity. Figure 9 shows that, only under the condition that turbulent $(\operatorname{Re}>2300)$ flow passes the superhydrophobic surface downstream of the backward-facing step, a stable large bubble would appear on the surface (Figure 9a), while large bubbles did not appear in laminar and transitional flow $(\operatorname{Re}<2300)$ (Figure 9 b).
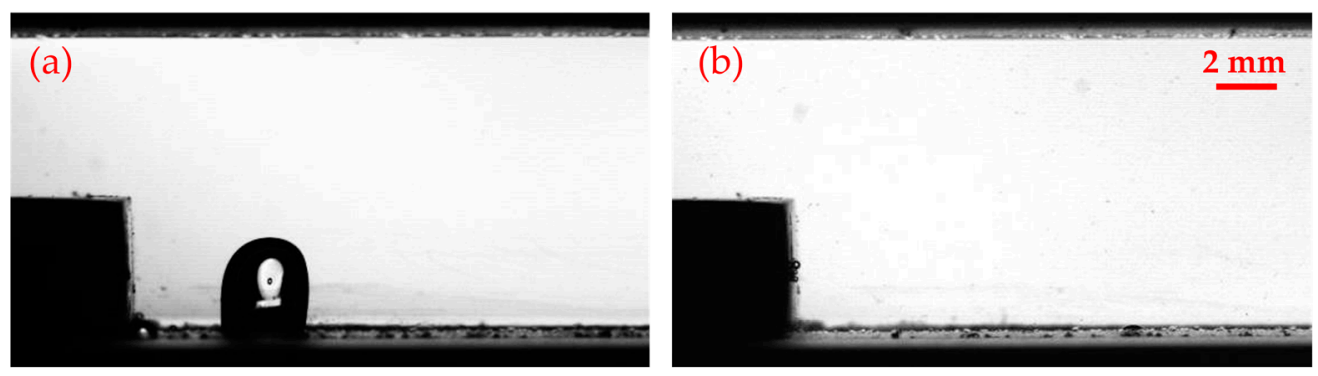

Figure 9. Comparison of laminar flow and turbulent flow. (a) $\operatorname{Re}>2300$; (b) $\operatorname{Re}<2300$.

Figure 10 shows the bubble attachment on the superhydrophobic surface at different Reynolds numbers beside the backward-facing step. It is worthwhile to note that the bubble is constantly changing with time, so, at each Reynolds number, the maximum volume and the closest hemispherical shape of the bubble are selected at the corresponding flow rate. It can be observed from the experiment that Reynolds number has large impacts on the size, position, shape, oscillation amplitude, detachment, and splitting of bubbles, which will be further analyzed below.

\subsection{Cause of Bubbles}

It can be seen from the experimental observation that there is a stable bubble on the superhydrophobic surface beside the backward-facing step, and the reason for the stable bubble in the liquid can only be boiling and cavitation. Under normal temperature conditions, boiling is impossible in the liquid, so the experimental observed phenomenon can only be cavitation. The cavitation is defined as [20] the gasification process that occurs when the local pressure in the liquid at room temperature decreases to the saturated vapor pressure of the liquid. In other words, cavitation is the formation, development, and collapse of gas bubbles inside or at the liquid-solid interface. 


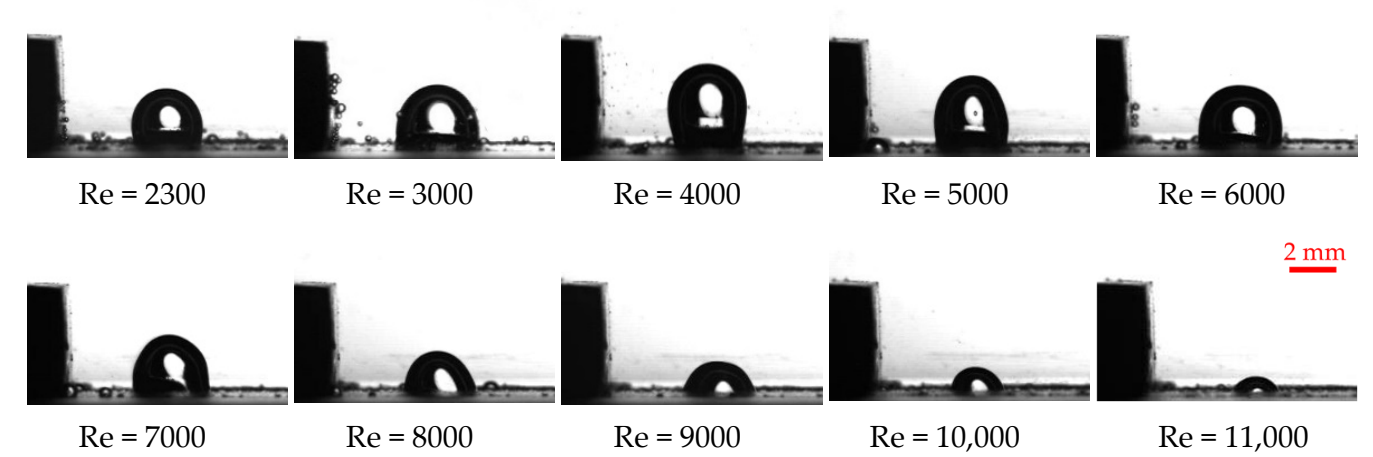

Figure 10. Bubble adhesion diagrams at different Reynolds numbers.

In order to verify the reliability of the experiments and to investigate the causes of the experimental phenomena, numerical simulations of the backward-facing step two-dimensional flow field have been performed using Fluent software. Based on the previous studies [21,22], the Reynolds averaging approach was chosen in this paper to simulate the turbulent flow field on the superhydrophobic surface downstream of the step. The pressure solver is selected, the SIMPLIC algorithm is chosen for the coupling of velocity and pressure, the second order format is chosen for the pressure discretization format, the second order upwind format is chosen for the momentum and turbulence discretization, and the convergence factors of pressure and momentum are kept as default. The standard $\mathrm{k}-\varepsilon$ model was used, the geometric model was the same as the dimensions of the test section, the bottom surface after the step was set as a slip surface, and the distribution of the pressure in the flow field of the backward-facing step was obtained, as shown in Figure 11. From the numerical simulation results, the minimum gauge pressure $p_{\min }$ of the flow field can be obtained (where $h$ is the height of the step, $x$ is the distance from the step, and $x / h$ is the dimensionless distance), as shown in Table 1 .

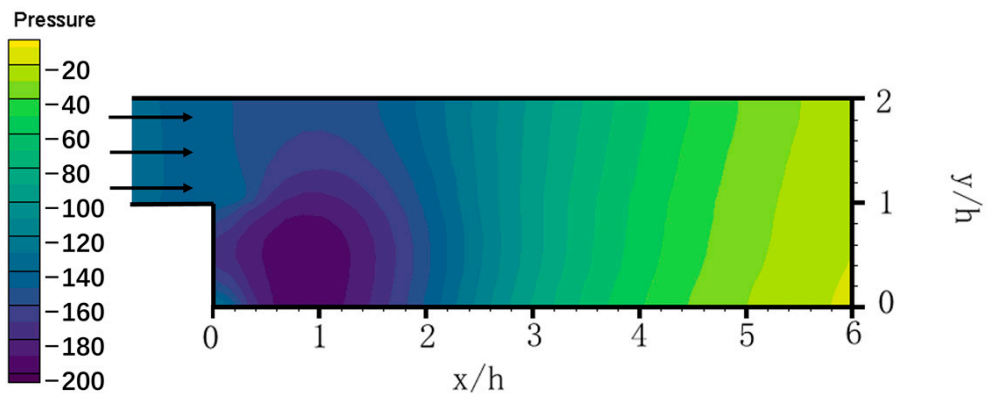

Figure 11. Pressure distribution when $\mathrm{Re}=4000$.

Table 1. The minimum gauge pressure in the flow field of the back step under different Reynolds numbers.

\begin{tabular}{ccccccccccc}
\hline $\boldsymbol{R e}$ & 2300 & 3000 & 4000 & 5000 & 6000 & 7000 & 8000 & 9000 & 10,000 & 11,000 \\
\hline$p_{\text {min }}(\mathbf{P a})$ & -53 & -88 & -155 & -239 & -341 & -460 & -596 & -750 & -922 & -1110 \\
\hline
\end{tabular}

It can be found from Table 1 that the minimum pressure in the backward-facing step flow field decreases with the increase of Reynolds number. It can be seen from the water saturated vapor pressure gauge that, when the experimental temperature is $20^{\circ} \mathrm{C}$, the saturated vapor pressure of water is $2338 \mathrm{~Pa}$. The minimum pressure (gauge pressure) in the experiment is $-1110 \mathrm{~Pa}$, that is, the absolute pressure is $100,215 \mathrm{~Pa}$, which is much higher than the saturated vapor pressure of water at $20^{\circ} \mathrm{C}$. Therefore, it is impossible to have phase transition in the channel. It can be known from the definition of cavitation that hydrodynamic cavitation cannot occur in the flow of the experimental pipeline. Anubhav Bhatt et al. [23] explored the cavitation phenomenon in the backward-facing step flow 
field through experiments, and the captured cavitation image is quite different from the bubble phenomenon in this experiment. The cavitation phenomenon in the experiment of Anubhav Bhatt et al. is not just a bubble; it is the formation and violent bursting of a large number of non-spherical bubbles. In this paper, only one hemispherical bubble appeared on the superhydrophobic surface after the step, and no violent rupture occurred. The comparison of the two experiments once again showed that the bubble generation in this experiment was not caused by hydraulic cavitation.

In fact, studies have shown that the occurrence of cavitation is not entirely dependent on pressure but also related to other factors. By means of a free-energy lattice Boltzmann simulation, G. Kähler et al. [24] found that the viscous stress, interfacial contributions to the local pressure, and the Laplace pressure are also relevant to the opening of a vapor cavity. In addition, G. Kahler et al. studied the formation process of the steam cavity below the corner of the bag wall when the fluid flows through the obstacle. This process is similar to the bubble formation process in this paper. In Reference [11] and this article, the pressure at the surface drops to a certain value, and the gas core at the wall evolves into a cavity over time. The exact shape and direction of the cavity growth depends on the pressure distribution in the channel. However, the difference is that the pressure near the wall in Reference [24] is lower than the equilibrium vapor pressure, and the gas in the cavity is steam. In this paper, the pressure at the wall is much higher than the equilibrium vapor pressure. The gas in the cavity comes from super hydrophobic surface and dissolved gases in the water. G. Falcucci et al. [25] also present direct evidence of flow-induced incipient cavitation through the lattice Boltzmann method. Therefore, in order to explore the causes of bubbles from the perspective of numerical simulation, a meso-micro method is required, such as the Lattice Boltzmann Method (LBM), which may be involved in future research.

According to the numerical simulation and experimental results, it is found that superhydrophobic surfaces and the backward-facing step provide the material basis and dynamic conditions for the occurrence of this phenomenon. The study of Brennan [20] (p. 16) shows that cavitation nuclei with a certain number and can stably exist in liquid, which poses the material conditions for liquid to form cavitation. And hydrophobic surfaces can lead to heterogeneous nucleation and greatly suppress the tensile strength of liquid. The stable bubble nucleon mechanism hypothesis proposed by Harvey $[26,27]$ suggests that the undissolved gas nuclei can stably exist in hydrophobic solid fractures because, in such cases, the surface tension will play a role in reducing pressure, so that the gas is not forced to dissolve and remains to be gas phase instead.

In the superhydrophobic surface of this experiment, there is a fabricated micro-nano structure (Figure 5), which provides material conditions for the generation of pseudocavitation. On the surface with these micro-nano structures, superhydrophobic coatings are also sprayed, so hydrophobic cracks are formed on the superhydrophobic surface and stable gas nuclei are found in the cracks, as shown in Figure 12. The generation of local low pressure in the liquid is the dynamic feature for this phenomenon. Due to the negative pressure downstream of the backward-facing step flow (Figure 11), the dissolved gas in the liquid continuously precipitates and gathers to the negative pressure area, and further diffuses into the tiny gas nuclei in the micro-nano structure on the superhydrophobic surface to make the bubbles continuously larger, forming a stable large bubble on the superhydrophobic surface. Thus, this phenomenon of bubble growth caused by the diffusion of dissolved gas in the liquid into the bubble through the mass transport of the bubble wall is named pseudo-cavitation, which can be categorized as non-phase-change cavitation [28] (pp. 7-10).

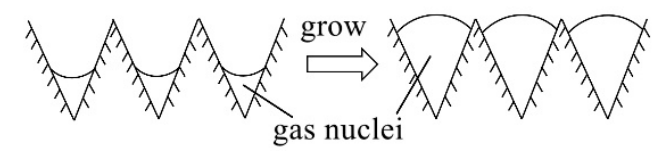

Figure 12. Schematic diagram of growth of gas nuclei in hydrophobic microstructure. 


\subsection{Evolution of Bubbles over Time}

Figure 13 show the whole process of bubble emergence, growth, oscillation, detachment, and splitting at the same Reynolds number as time changes, which can be defined as a bubble cycle. In a bubble cycle, firstly, the gas nucleus is generated in the micro-nano structure cracks on the superhydrophobic surface, as shown in growth process in Figure 13. Then, under the action of negative pressure, the non-condensable gas in the water precipitates and diffuses into the tiny gas nucleus between the cracks, resulting in the continuous growth of bubbles, as shown in Figure 13. During the growth process, the bubbles continuously oscillate and deform on the surface, and the frequency of oscillation is very high, which increases the disturbance of the flow field. After a period of development, the bubble volume reached the maximum. In the small Reynolds number stage $(\operatorname{Re}<4000)$, bubbles adhere to the micro-nano structure superhydrophobic surface stably. With the increase of Reynolds number, bubbles detached from the wall. Because of the separation vortex in the recirculating flow region, the bubbles firstly move to the left and then separate along the streamline trajectory of the separation vortex. As shown in Figure 13, the bubble leaves gas nucleus in its original position after detachment, creating conditions for bubble regrowth After the bubbles are detached, they are torn in the liquid and split. Then, bubbles grow again on the surface, and the next bubble cycle begins. In a bubble cycle, the detachment and splitting of the bubbles are very fast, occurring in the last second, and the bubbles are growing and oscillating at other times.

\subsection{Effect of Reynolds Number on Bubble Position and Size}

The pressure distribution in the flow field at different Reynolds numbers can be obtained from the numerical simulation results. The pressure distribution not only affects the position of bubble formation but also affects the growth of gas nuclei. Figure 14 shows the pressure distribution on the superhydrophobic surface downstream of the backwardfacing step at different Reynolds numbers. As shown in Figure 14, with the change of position, the pressure on the surface decreases first and then increases. There is a minimum pressure value at a certain point, where the tensile strength of the liquid is the weakest in the liquid. The gas nucleus attached to this position on the superhydrophobic surface expands gradually under the negative pressure, tearing the liquid and growing into large bubbles.

As shown in Figure 14, on the superhydrophobic surface downstream of the backwardfacing step, the minimum pressure gradually decreases with the increase of Reynolds number. The position of the lowest pressure point shows the following law with the change of Reynolds number. When the Reynolds number increases from 2300 to 6000, the position of the lowest pressure on the surface gradually moves away from the backward-facing step. When the Reynolds number increases from 6000 to 9000, the position of the minimum pressure on the surface is fixed at $x / h=1.176$ and does not change. With the Reynolds number increasing from 9000 to 11000 , the position of the lowest pressure on the surface is gradually away from the step. In the simulation results, the trend of the position of the lowest pressure point on the superhydrophobic surface with the Reynolds number provides the underlying reason for the trend of the bubble emerging position.

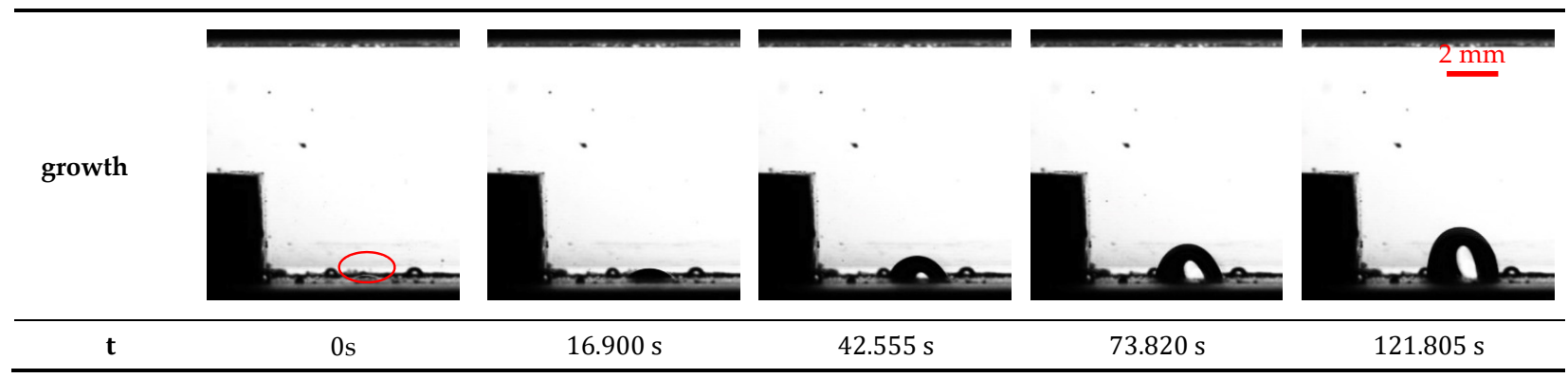

Figure 13. Cont. 


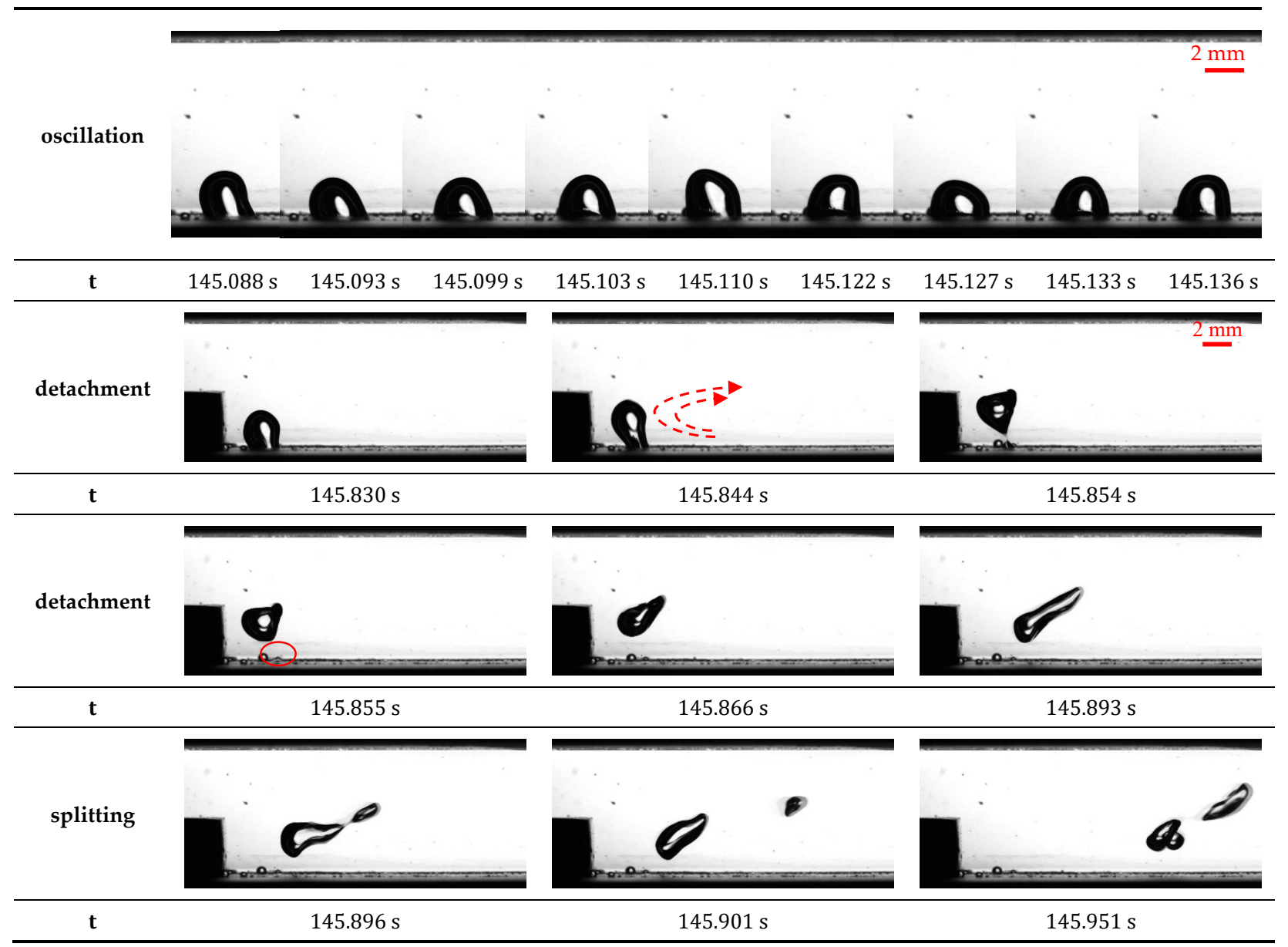

Figure 13. A bubble cycle when $\operatorname{Re}=7000$ and bubble dynamics.

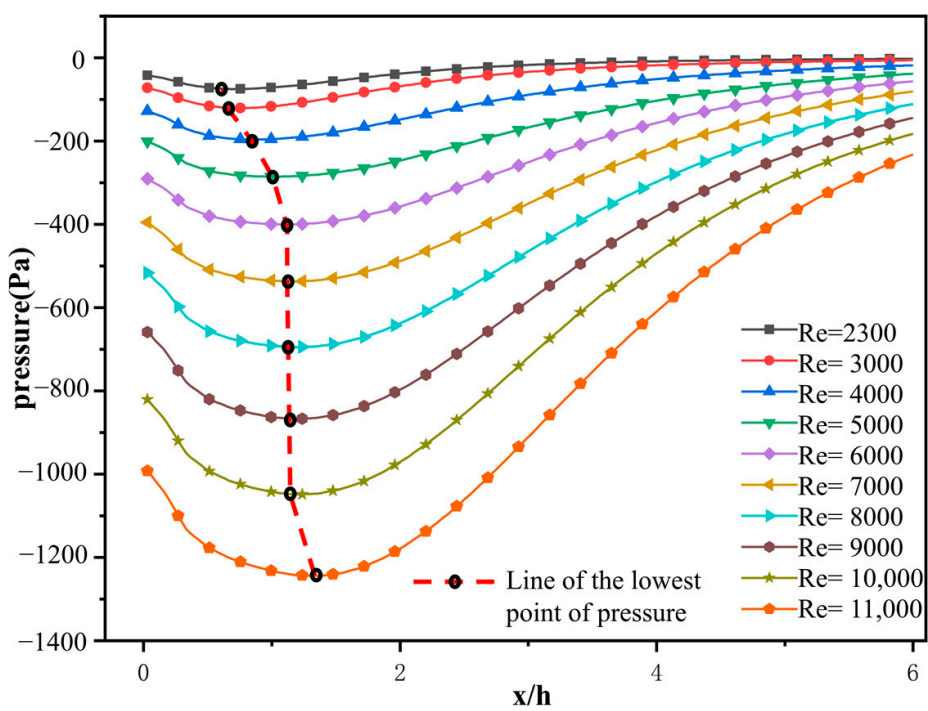

Figure 14. Pressure distribution at different Reynolds numbers.

It is found through experiments that, besides the fact that the gas nuclei at the lowest pressure point on the superhydrophobic surface can grow into bubbles, the gas nuclei in other low-pressure regions near this point may also develop into bubbles, as shown in Figure 15. However, the bubbles generated in the low-pressure region near the lowest pressure point are not stable, which will quickly approach the bubbles at the lowest 
pressure point under the driving force of pressure difference. Finally, the bubbles merge into a bubble at the lowest pressure point with large volume.

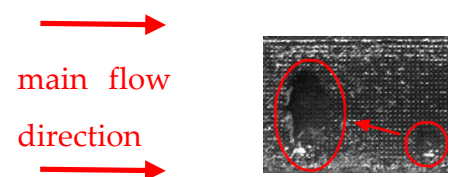

$\mathrm{t}=0 \mathrm{~ms}$

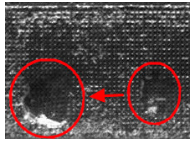

$\mathrm{t}=5 \mathrm{~ms}$

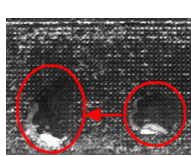

$\mathrm{t}=10 \mathrm{~ms}$

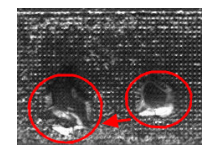

$\mathrm{t}=15 \mathrm{~ms}$

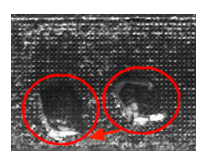

$\mathrm{t}=20 \mathrm{~ms}$

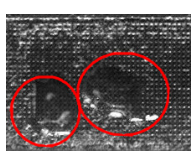

$\mathrm{t}=25 \mathrm{~ms}$

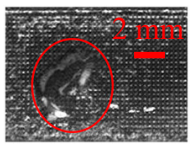

$\mathrm{t}=30 \mathrm{~ms}$

Figure 15. Top view when $\operatorname{Re}=11,000$.

Figure 16 shows the change of the bubble centroid position (Center-X) with Reynolds number in the experiment. It is found that the bubble position does not change significantly and does not show certain regularity. This is due to the small size of the experimental pipe, the small range of variation of the bubble position, and the fact that the measured data of the bubble center-of-mass position is an average value, resulting in the inability to accurately measure the variation pattern of the bubble position in the experiment. Meanwhile, Figure 16 indicates the variation of the equivalent volume diameter $\left(d_{e}\right)$ of bubbles in the backward-facing step flow field at different Reynolds numbers. When the Reynolds number increases from 2300 to 4000 , the average equivalent volume diameter of bubbles increases rapidly from $2.82 \mathrm{~mm}$ to $3.13 \mathrm{~mm}$. When Reynolds number increases from 4000 to 7000 , the average equivalent volume diameter of bubbles decreases from $3.13 \mathrm{~mm}$ to $2.70 \mathrm{~mm}$, and the change rate is slow. When the Reynolds number increases from 7000 to 11,000 , the average equivalent volume diameter of the bubble decreases sharply from $2.70 \mathrm{~mm}$ to $1.28 \mathrm{~mm}$, and the bubble volume decreases rapidly.

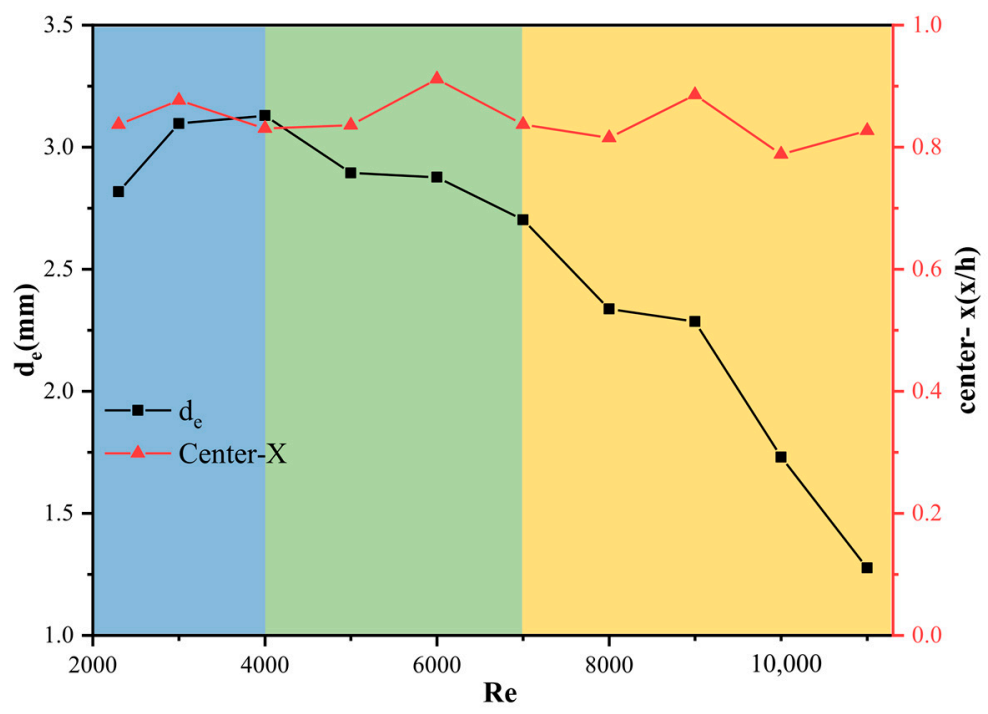

Figure 16. The position and size of the bubble changes.

As shown in Figure 17, the flow field and the distribution of bubble behind the step are shown. Qi et al. [29] found that, when $2000<\operatorname{Re}<3500$, the length $L_{r}$ of the recirculating flow region increases with the increase of Reynolds number, and when $\operatorname{Re}>3500$, the length $L_{r}$ of the recirculating flow region is independent of Reynolds number and tends to a stable value. Therefore, in this experiment, when $2300<\operatorname{Re}<4000$, with the increase of Reynolds number, the length of the recirculating flow region $L_{r}$ also increases, and the separation line changes from separation line II to separation line I. Influenced by the height of the separation line, the maximum volume of the bubble under the corresponding Reynolds number becomes larger with the increase of Reynolds number. When $\operatorname{Re}>4000$, with the increase of Reynolds number, the length $L_{r}$ of the recirculating flow region no 
longer changes, and the position of the separation line no longer changes, but the reflux velocity of the recirculating flow region is increasing, and the impact on the bubble is getting bigger and bigger, which leads to the maximum volume of the bubble formed at the corresponding Reynolds number getting smaller and smaller.

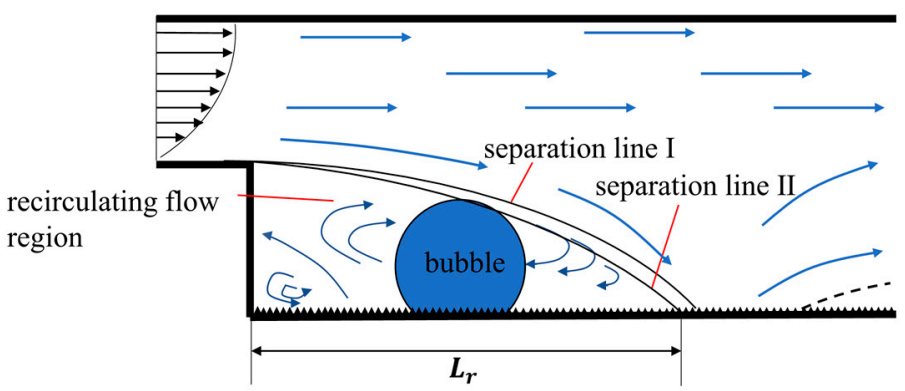

Figure 17. Schematic diagram of the flow field in which the bubbles are located.

\subsection{Effect of Reynolds Number on Bubble Oscillation Amplitude and Shape}

The deformation of bubbles is studied by the aspect ratio $E$ analysis, as shown in Figure 18. When the bubble volume no longer increases at each Reynolds number, the data of bubble aspect ratio varying with time is selected for analysis. It can be seen from Figure 18 that, when the Reynolds number is 2300 , the disturbance of water flow is small, and the aspect ratio of bubbles is about 0.77 and remains almost unchanged, indicating that the oscillation amplitude of bubbles is small. When the Reynolds number increases, the flow disturbance becomes larger, and the aspect ratio curve of bubbles begins to oscillate. With the increase of Reynolds number, the oscillation amplitude of the bubble aspect ratio curve increases, showing that, with the increase of Reynolds number, the oscillation amplitude of the bubble due to the influence of water flow disturbance increases, and its deformation rate also increases. When the Reynolds number is 11,000 , the volume of the bubble is small, so the oscillation amplitude is less affected by the flow disturbance. It can be seen from Figure 18 that the aspect ratio change under this condition is relatively small.

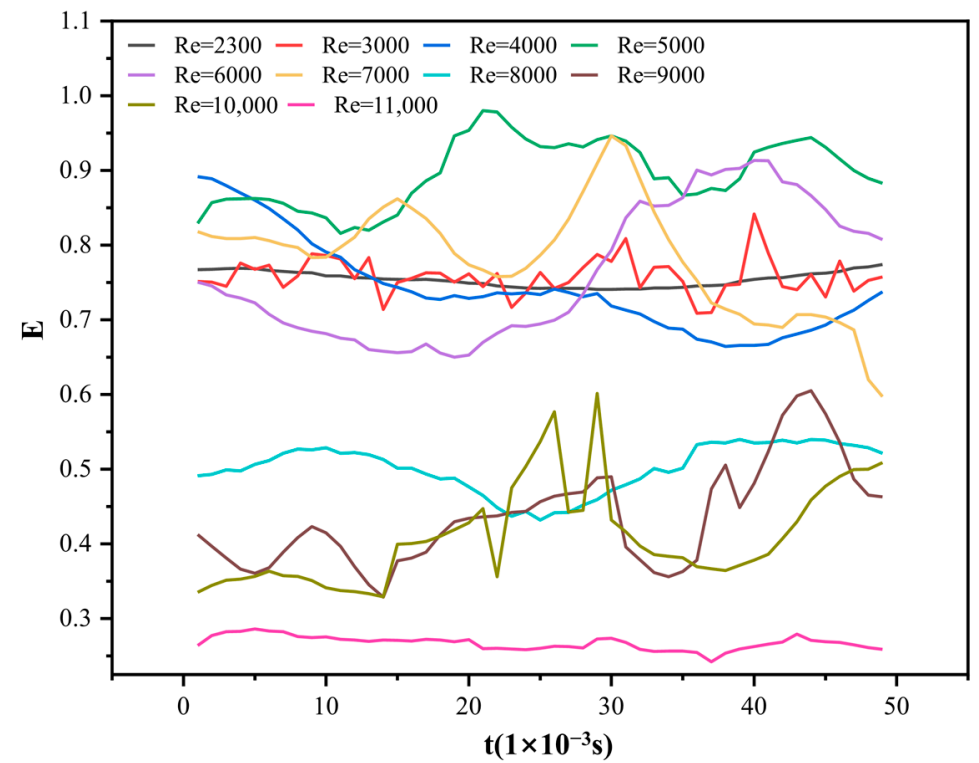

Figure 18. The changing law of bubble aspect ratio.

When the Reynolds number is between 2300 and 7000, the aspect ratio varies from 0.65 to 1 , indicating that the bubble shape is close to spherical cap shape at this stage. When Reynolds number is between $8000 \sim 10,000$, the aspect ratio decreases, the height of 
the bubble is $0.3 \sim 0.6$ times of the width, and the shape of the bubble is close to elliptical cap shape. When the Reynolds number is 11,000 , the aspect ratio of the bubble further decreases, indicating that the bubble is flatter. It can be directly seen from Figure 19.

\begin{tabular}{|c|c|c|c|c|c|c|c|c|c|c|}
\hline $\begin{array}{l}\text { Size and } \\
\text { shape of } \\
\text { bubbles }\end{array}$ & & & & & & Q & & & n & $\begin{array}{l}2 \mathrm{~mm} \\
\simeq\end{array}$ \\
\hline $\operatorname{Re}$ & 2300 & 3000 & 4000 & 5000 & 6000 & 7000 & 8000 & 9000 & 10,000 & 11,000 \\
\hline
\end{tabular}

Figure 19. The shape of bubbles under different Reynolds numbers.

\subsection{Effect of Reynolds Number on Bubble Detachment and Splitting}

Bubble detachment also follows a certain regularity with the change of Reynolds number, which can be divided into two regions according to the trend shown in Figure 20. For the region without detachment phenomenon (Reynolds number between 2300 5000), the bubbles stably adhere to the superhydrophobic surface downstream of the step, and there is no bubble detachment and splitting. For the region with detachment phenomenon (Reynolds number between 5000 11,000), when the Reynolds number is 5000, the bubbles begin to detach, split, and grow again, with an average bubble cycle of $315 \mathrm{~s}$. When the Reynolds number further increases from 5000 to 6000, the bubble period is getting smaller and smaller, and the reduction rate is fast. Then, when the Reynolds number is between 6000 and 11,000, with the increase of Reynolds number, the frequency of bubbles from the wall is getting faster and faster, and the bubble period is gradually decreasing, while the reduction rate is slower.

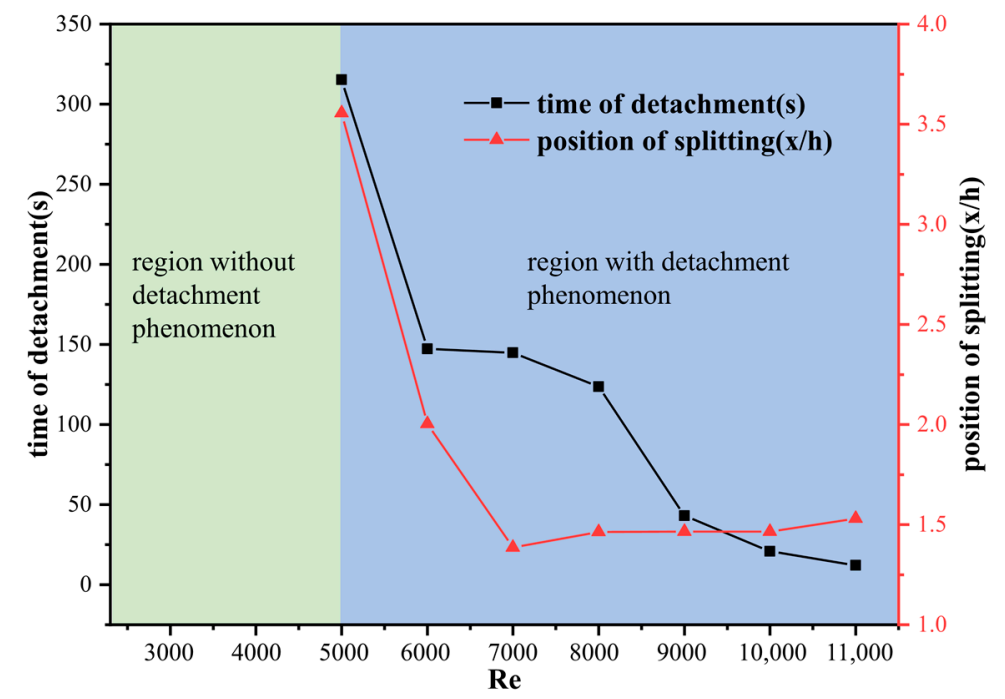

Figure 20. Detachment time and splitting location of bubbles.

Figure 20 shows the variation of bubble splitting position with Reynolds number. It can be seen from the figure that the position range of bubble splitting is $x / h=1.3 \sim 3.6$. With the Reynolds number increasing from 5000 to 7000 , the bubble splitting position is closer to the step. When the Reynolds number increases from 7000 to 10,000 , the splitting position of the bubble is basically unchanged and stable at $x / h=1.5$.

By extracting the bubble centroid position, the trajectory of the bubble from detaching the surface to before splitting is obtained, as shown in Figure 21. According to the statistical results of the bubbles from the surface to the splitting and the experimental observations after the bubbles splitting, bubble trajectory I and bubble trajectory II in Figure 22 can be drawn. Figure 22 is the typical flow structure of backward-facing step flow. The flow field 
downstream of the step can be divided into separation region, reattachment region, and redevelopment region along the flow direction. The flow field downstream of the step can be divided into corner vortex region, recirculating flow region, shear layer region, and mainstream region along the vertical direction [30].

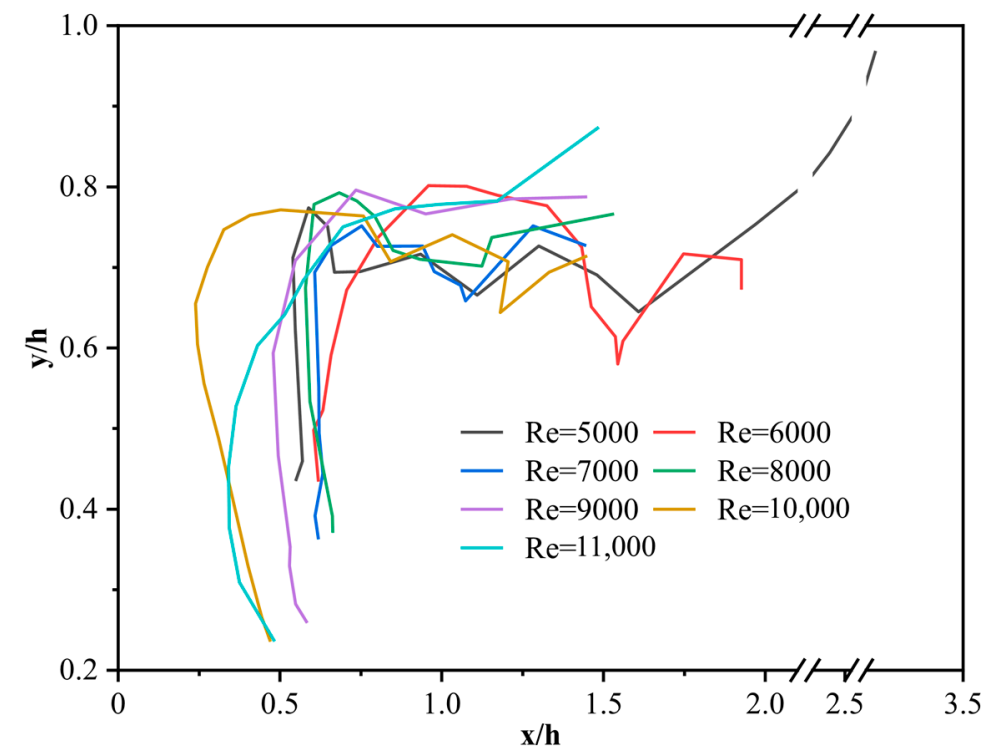

Figure 21. Motion trajectory diagram after the bubble is separated.

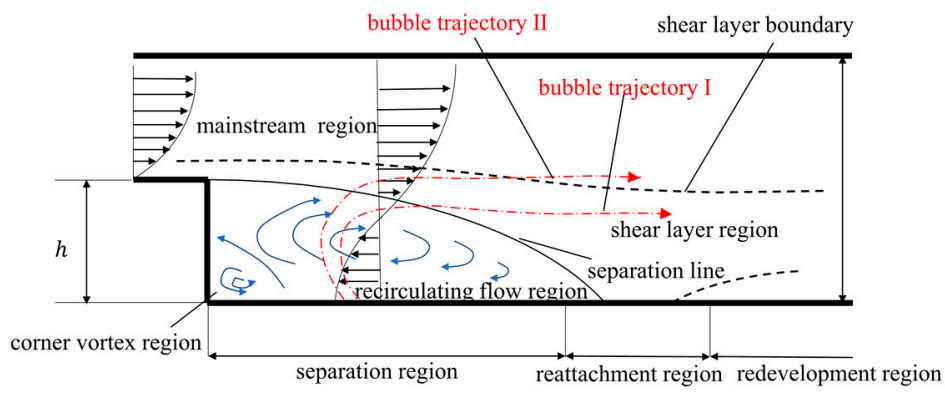

Figure 22. Typical flow structure of backward-facing step flow.

It can be seen from Figure 13 that, when bubbles split, multiple bubbles will be split from the separated large bubbles, and the reason for its split can be explained by combining Figures 21 and 22. As shown in Figure 22, after the bubble departs from the surface, it firstly moves along the trajectory of the separation vortex in the recirculating flow region, then passes through the partition line of the recirculating flow region and the shear layer region along the bubble trajectory I, and moves from the recirculating flow region to the shear layer region, and, finally, moves downstream. Another possible is the bubble moves along the bubble trajectory II from the recirculating flow region through the separation line to the shear layer region, and then through the shear layer boundary to the mainstream region. When bubbles pass through the separation line or the shear layer boundary, the bubbles are split into multiple bubbles by the separation line or the shear layer boundary due to the different fluid velocities in each region. When the Reynolds number is between 5000 7000, the volume of the detached bubble is larger, and the buoyancy force is greater, so the trajectory of the detached bubble is bubble trajectory II. And, when the bubble splits, the larger bubble moves along the bubble trajectory II and the smaller bubble moves along the bubble trajectory I. When the Reynolds number is between 7000 11,000, the volume of the detached bubble is smaller, and the buoyancy force is smaller, so the trajectory of the detached bubble is bubble trajectory I. 


\section{Conclusions}

In this paper, in the process of exploring the influence of superhydrophobic surface on the turbulent flow field, we found the pseudo-cavitation phenomenon on the superhydrophobic surface in the backward-facing step turbulent flow field. In order to reveal the fundamental mechanisms for this phenomenon, this study analyzes the causes of bubble formation, the variation of bubble with time under the same Reynolds number and the influence of Reynolds number on the position, size, oscillation amplitude, shape, detachment, and splitting of bubbles. The conclusions are as follows.

1. The superhydrophobic surface provides the material basis for the generation of pseudo-cavitation phenomenon, and the gas nuclei can stabilize the hydrophobic microstructure cracks. Then, the backward-facing step structure provides dynamic conditions for the generation of pseudo cavitation. Backward-facing step structure creates a low-pressure area downstream of the step flow field, which provides a low-pressure environment for the growth of gas nuclei.

2. Pseudo-cavitation on superhydrophobic surface occurs at Reynolds number between 2300 and 11,000. When Reynolds number is less than 4000, the growth and oscillation of bubbles appear with the change of time. When Reynolds number is greater than 4000 , the growth, oscillation, detachment, splitting, and regrowth of bubbles appear with the change of time.

3. In the mainstream direction, the pressure on the superhydrophobic surface downstream of the step decreases firstly and then increases, and there is a minimum pressure point. The position where the pressure is the smallest is the position where the bubble initiates and grows. The location with the smallest pressure gradually moves away from the backward-facing step with the increase of Reynolds number, but this change is quite small. When Reynolds number increases from 2300 to 4000, the bubble volume becomes larger, while the bubble volume becomes smaller when Reynolds number increases from 4000 to 11,000 .

4. With the increase of Reynolds number, the oscillation amplitude of bubbles increases, and the shape of bubbles becomes flatter.

5. With the increase of Reynolds number, the bubble detachment time is smaller, and the bubble splitting position is closer to the backward-facing step.

By exploring the causes of bubble generation and development laws in the pseudocavitation phenomenon, it is important to better understand the flow mechanism and gas coverage of superhydrophobic surface under condition of backward-facing step, as well as paves the way for studying the flow resistance reduction effect on superhydrophobic surfaces. Besides, it can be used to better control the perturbation of the flow field. Meanwhile, the finding of this phenomenon brings great inspiration to the study of superhydrophobic surface. The rational design of steps and superhydrophobic surface structures on the surface to achieve both resistance reduction and enhanced heat transfer is the focus of future research.

Author Contributions: Conceptualization, X.L. and Y.L.; methodology, X.L. and J.L.; software, W.-T.W.; validation, K.M.; data curation, L.G.; writing-original draft preparation, X.L. and Y.L.; funding acquisition, Y.L. and J.L. All authors have read and agreed to the published version of the manuscript.

Funding: This research was funded by the Fundamental Research Funds for the Central Universities, China (DUT20RC (3)095, DUT20JC21) and National Natural Science Foundation of China, China (Grant No. 51876027, 51806028).

Institutional Review Board Statement: Not applicable.

Informed Consent Statement: Not applicable.

Data Availability Statement: The data presented in this study are available on request from the corresponding author. The data are not publicly available due to privacy restrictions. 
Conflicts of Interest: The authors declare no conflict of interest.

\section{References}

1. Gao, X.; Yan, X.; Yao, X.; Xu, L.; Zhang, K.; Zhang, J.; Yang, B.; Jiang, L. The Dry-Style Antifogging Properties of Mosquito Compound Eyes and Artificial Analogues Prepared by Soft Lithography. Adv. Mater. 2007, 19, 2213-2217. [CrossRef]

2. Young, T. An Essay on the Cohesion of Fluids. R. Soc. 1805, 95, 65-87.

3. Wenzel, R.N. Resistance of Solid Surfaces to Wetting by Water. Ind. Eng. Chem. 1936, 28, 988-994. [CrossRef]

4. Cassie, A.B.D.; Baxter, S. Wettability of porous surfaces. Trans. Faraday Soc. 1944, 40, 546. [CrossRef]

5. Bittoun, E.; Marmur, A. Optimizing Super-Hydrophobic Surfaces: Criteria for Comparison of Surface Topographies. J. Adhes. Sci. Technol. 2009, 23, 401-411. [CrossRef]

6. Patankar, N.A. Mimicking the Lotus Effect: Influence of Double Roughness Structures and Slender Pillars. Langmuir 2004, 20, 8209-8213. [CrossRef]

7. Wang, S.; Jiang, L. Definition of Superhydrophobic States. Adv. Mater. 2007, 19, 3423-3424. [CrossRef]

8. Blossey, R. Self-cleaning surfaces-Virtual realities. Nat. Mater. 2003, 2, 301-306. [CrossRef]

9. Park, H.; Sun, G.; Kim, C.C. Superhydrophobic turbulent drag reduction as a function of surface grating parameters. J. Fluid Mech. 2014, 747, 722-734. [CrossRef]

10. Wang, B.; Wang, J.; Dou, Z.; Chen, D. Investigation of retention of gases in transverse hydrophobic microgrooved surfaces for drag reduction. Ocean Eng. 2014, 79, 58-66. [CrossRef]

11. Martell, M.B.; Perot, J.B.; Rothstein, J.P. Direct numerical simulations of turbulent flows over superhydrophobic surfaces. J. Fluid Mech. 2009, 620, 31-41. [CrossRef]

12. Cowley, A.; Maynes, D.; Crockett, J. Inertial effects on thermal transport in superhydrophobic microchannels. Int. J. Heat Mass Transf. 2016, 101, 121-132. [CrossRef]

13. Fuaad, P.A.; Arul Prakash, K. Influence of texture on thermal transport in streamwise-aligned superhydrophobic turbulent channels. Int. J. Therm. Sci. 2017, 114, 72-85. [CrossRef]

14. Wang, F.; Gao, A.; Wu, S.; Zhu, S.; Dai, J.; Liao, Q. Experimental Investigation of Coherent Vortex Structures in a Backward-Facing Step Flow. Water 2019, 11, 2629. [CrossRef]

15. Qi, C.; Ding, Z.; Tu, J.; Wang, Y.; Wang, Y. Study on backward-facing step flow and heat transfer characteristics of hybrid nanofluids. J. Therm. Anal. Calorim. 2020. [CrossRef]

16. Liu, L.; Yan, H.; Zhao, G. Experimental studies on the shape and motion of air bubbles in viscous liquids. Exp. Therm. Fluid Sci. 2015, 62, 109-121. [CrossRef]

17. Aoyama, S.; Hayashi, K.; Hosokawa, S.; Tomiyama, A. Shapes of ellipsoidal bubbles in infinite stagnant liquids. Int. J. Multiphas. Flow 2016, 79, 23-30. [CrossRef]

18. Dijkhuizen, W.; van Sint Annaland, M.; Kuipers, J.A.M. Numerical and experimental investigation of the lift force on single bubbles. Chem. Eng. Sci. 2010, 65, 1274-1287. [CrossRef]

19. Reilly Meehan, R.; Donnelly, B.; Nolan, K.; Persoons, T.; Murray, D.B. Flow structures and dynamics in the wakes of sliding bubbles. Int. J. Multiphas. Flow 2016, 84, 145-154. [CrossRef]

20. Brennen, C.E. Cavitation and Bubble Dynamics; Oxford University Press: New York, NY, USA, 1995.

21. Frendi, A.; Harrison, C. Partially Averaged Navier-Stokes: A (k- $\omega) /(k-\varepsilon)$ Bridging Model. Fluids 2020, 5, 129. [CrossRef]

22. Kelly, N.S.; Gill, H.S.; Cookson, A.N.; Fraser, K.H. Influence of Shear-Thinning Blood Rheology on the Laminar-Turbulent Transition over a Backward Facing Step. Fluids 2020, 5, 57. [CrossRef]

23. Bhatt, A.; Ganesh, H.; Ceccio, S.L. Cavitating flow behind a backward facing step. Int. J. Multiphas. Flow 2021, $139,103584$. [CrossRef]

24. Kähler, G.; Bonelli, F.; Gonnella, G.; Lamura, A. Cavitation inception of a van der Waals fluid at a sack-wall obstacle. Phys. Fluids 2015, 27, 123307. [CrossRef]

25. Falcucci, G.; Jannelli, E.; Ubertini, S.; Succi, S. Direct numerical evidence of stress-induced cavitation. J. Fluid Mech. 2013, 728, 362-375. [CrossRef]

26. Harvey, E.N.; Barnes, D.K.; McElroy, W.D.; Whiteley, A.H.; Pease, D.C.; Cooper, K.W. Bubble formation in animals. I. Physical factors. J. Cell. Comp. Physiol. 1944, 24, 1-22. [CrossRef]

27. Harvey, E.N.; McElroy, W.D.; Whiteley, A.H. On Cavity Formation in Water. J. Appl. Phys. 1947, 18, 162-172. [CrossRef]

28. Sensen, P.; Xiaoxing, P. Physical Mechanism of Cavitation; National Defense Industry Press: Beijing, China, 2013 ; p. 181.

29. Qi, E.; Huang, M.; Li, W.; Zhang, X. A Experimental Study on the 2D Time-average Flow over a Backward Facing Step via PIV. J. Exp. Mech. 2006, 21, 225-232.

30. Armaly, B.F.; Durst, F.; Pereira, J.C.F.; Schönung, B. Experimental and theoretical investigation of backward-facing step flow. J. Fluid Mech. 1983, 127, 473. [CrossRef] 